\title{
3 Research Square

\section{Characterization of a potential probiotic bacterium Lactococcus raffinolactis WiKim0068 isolated from fermented vegetable using genomic and in vitro analyses}

Min Young Jung

World Kimchi Institute

Changsu Lee

World Kimchi Institute

Myung-Ji Seo

Incheon National University

Se Hee Lee

World Kimchi Institute

Seong Woon Roh ( $\square$ seong18@gmail.com )

World Institute of Kimchi https://orcid.org/0000-0003-0609-6130

\section{Research article}

Keywords: Lactococcus raffinolactis, genome sequence, kimchi, probiotics, vitamin B3

Posted Date: March 3rd, 2020

DOI: https://doi.org/10.21203/rs.2.19681/v2

License: (c) (1) This work is licensed under a Creative Commons Attribution 4.0 International License.

Read Full License

Version of Record: A version of this preprint was published at BMC Microbiology on May 27th, 2020. See the published version at https://doi.org/10.1186/s12866-020-01820-9. 


\section{Abstract}

Background: Lactococcus members belonging to lactic acid bacteria are widely used as starter bacteria in the production of fermented dairy products. From kimchi, a Korean food made of fermented vegetables, Lactococcus raffinolactis WiKim0068 was isolated and its genome was analyzed. Results: The complete genome of the strain WiKim0068 consists of one chromosome and two plasmids that comprises 2,292,235 bp, with a G+C content of $39.7 \mathrm{~mol} \%$. Analysis of orthoANI values among Lactococcus genome sequences showed that the strain WiKim0068 has $>67 \%$ sequence similarity to other species and subspecies. In addition, it displayed no antibiotic resistance and can metabolize nicotinate and nicotinamide (vitamin B3). Conclusion: These results augments our understanding of the genus Lactococcus and suggest that this new strain has potential industrial applications. Keywords: Lactococcus raffinolactis, genome sequence, kimchi, probiotics, vitamin B3

\section{Background}

Lactic acid bacteria (LAB) activity improves the texture, flavor, and scent of dairy products during fermentation and ripening [1]. In these products, LAB starters contribute to flavor development through the (bio)chemical conversion of milk components, such as lactose, fat, casein via glycolysis, lipolysis, and proteolysis, respectively [2]. Moreover, another favorable property of LAB is its ability to adhere to the host intestinal tract which enables them to be effective probiotic strains [3].

The genus Lactococcus includes Gram-positive, catalase-negative, non-motile, non-sporulating, coccishaped LAB [4]. Lactococcus members are widely used as starter bacteria in the production of fermented dairy products, such as cheese and yogurt $[5,6]$. Three species within the genus Lactococcus $-L$. raffinolactis, $L$. lactis subsp. lactis, and $L$. lactis subsp. cremoris-are listed among the inventory of microbial food cultures (MFC) of fermented food products as species with demonstrated safety [7].

L. raffinolactis is distributed in a wide range of products, including fermented foods such as fish, meat, vegetables, and milk and other materials of plant and animal origin [8, 9]. L. raffinolactis, can ferment agalactosides, such as raffinose and melibios, which are not used by L. lactis [10,11]. This feature is a significant advantage for use as a starter in dairy products. In this study, we report the isolation, identification, and characterization of the $L$. raffinolactis WiKim0068 isolated from fermented cabbage (kimchi). We also evaluated the possibility of using the strain WiKim0068 in dairy products, and the safety of the strain. Further, we analyzed its proteolytic enzymes through complete genome sequence analysis. In vitro assays and predictive gene analysis for antibiotic resistance and adhesion were also performed.

\section{Results And Discussion}

\section{Phylogenetic and phenotypic features of the isolated LAB strain}


The bacterial strain, designated WiKim0068, was isolated from a Korean fermented food, kimchi. In order to identify the phylogenetic similarity of the strain, 16S rRNA gene based phylogenetic analysis of strain WiKim0068 was performed and the closely related strains were found to be $L$. raffinolactis NBRC $100932^{\top}$ with a similarity of $99.9 \%$ (Fig. 1). This result indicated that strain WiKim0068 belongs to $L$. raffinolactis species. Sugar assimilation/acid formation test conducted using API $50 \mathrm{CH}$ revealed positive results for galactose, glucose, fructose, mannose, mannitol, $N$-acetylglucosamine, esculin, ferric citrate, salicin, cellobiose, maltose, melibiose, saccharose, trehalose, raffinose, and turanose, whereas $\mathrm{H}_{2} \mathrm{~S}$ production and urease were negative. Enzyme detection performed with an API ZYM kit indicated esterase, leucine arylamidase, and naphthol-AS-BI-phosphohydrolase activities.

\section{General genomic features of $L$. raffinolactis WiKim0068}

The PacBio RS II sequencing system generated 74,558 reads, with an average read length of $8,212 \mathrm{bp}$. The complete genome of the strain WiKim0068 consisted of a circular $2.22 \mathrm{Mb}$ chromosome and two circular plasmids, with a total size of $2.29 \mathrm{Mb}$. The chromosome contained 2,060 predicted proteincoding genes (CDSs), 13 rRNA genes (5S rRNA, 5; 16S rRNA, 4; 23 S rRNA, 4), 55 tRNAs, and 3 other RNAs. The WiKim0068 genome was found to contain $39.7 \mathrm{~mol} \% \mathrm{G}+\mathrm{C}$ content (Table 1), which is within the range of $35.5-46.4 \%$ reported for Lactococcus species [12], and similar to the $39.25 \mathrm{~mol} \%$ observed in two L. raffinolactis strains, 4877 (CALL00000000) and NBRC $100932^{\top}$ (BCVN00000000). For functional classification, WiKim0068 genome was analyzed using the clusters of orthologous genes (COG) database (http://www.ncbi.nlm.nih.gov/COG/), and 2,000 genes were annotated. The annotated genes were associated with the following categories: general function prediction only ( $R ; 237$ genes), carbohydrate transport and metabolism (G; 210 genes), function unknown (S; 190 genes), amino acid transport and metabolism ( $\mathrm{E} ; 180$ genes), coenzyme transport and metabolism $(\mathrm{H} ; 70$ genes), defense mechanisms ( $\mathrm{V} ; 63$ genes), and secondary metabolites biosynthesis, transport, and catabolism (Q; 15 genes; Supplementary Table S1). In addition, Rapid Annotation using Subsystem Technology analysis revealed genes related to the following categories: stress response $(2.62 \%)$, cofactors, vitamins, prosthetic groups, pigments (5.30\%), and virulence, disease, and defense (3.39\%) (Supplementary Fig. S1). Stress response-related genes category included: "osmotic stress" (5 genes), "oxidative stress" (17 genes), "cold shock" (1 gene), "heat shock" (15 genes), "detoxification" (9 genes), and "no subcategory" (1 gene). The category of cofactors, vitamins, prosthetic groups, pigments included those related to "biotin" (15 genes), "NAD and NADP" (14 genes), "riboflavin, FMN, FAD" (8 genes), and "folate and pterines" (33 genes). The category of virulence, disease, and defense included mainly those involved in "bacteriocins, ribosomally synthesized antibacterial peptides" (8 genes), "resistance to antibiotics and toxic compounds" (29 genes), and "invasion and intracellular resistance" (15 genes). Of these, biotin, riboflavin, and folate are related to human health and digestion and cause various symptoms when deficient [13]. Bacteriocins are antimicrobial peptides produced by bacteria [14] and an alternative to treat antibiotic resistant bacteria. Significantly, bacteriocins production have been regarded as an important feature in the selection of probiotic strains. These genes were associated with the presence of useful probiotic characteristics, which play important roles in the food and pharmaceutical industries [15-17]. 


\section{Comparative genomic analysis}

Analysis of the orthologous average nucleotide identity (orthoANI) values among Lactococcus genome sequences showed that strain WiKim0068 had 68.55-98.73\% genome sequence similarities with other species and subspecies. Its genome was most closely related to that of L. raffinolactis NBRC $100932^{\top}$ (98.73\%), followed by L. raffinolactis 4877 (87.02\%), L. piscium MKFS47 (76.57\%), L. lactis subsp. lactis IL 1403 (69.41\%), L. fujiensis JCM 16395 (68.12\%), and L. garvieae ATCC 49156 (68.55\%; Fig. 2). Thus, the comparative whole genome sequence analysis indicated that the strain WiKim0068 belongs to the species $L$. raffinolactis [18]. Its similarity to its two closest relative strains ( $L$. raffinolactis NBRC $100932^{\top}$ and $L$. raffinolactis 4877), based on BLAST comparison, is shown in Fig. 3 . This figure describes the difference in GC contents and similarities between strain WiKim0068 and two closest relative strains. Furthermore, we searched for clustered regularly interspaced short palindromic repeats (CRISPRs) using the CRISPRFinder platform, but no confirmed CRISPRs were found in the WiKim0068 genome.

\section{Phage and pathogenesis-related genes}

PHAST analysis was performed to identify prophage contamination in the genome of WiKim0068. The chromosome contained two intact, one incomplete, and one questionable prophage. The first plasmid (pWiKim0068-1) contained only one incomplete prophage, while the second plasmid (pWiKim0068-2) contained none (Supplementary Fig. S2). Intact prophage regions were located between positions $57,319-90,123$ and $1,524,268-1,563,900$ bp of the chromosome.

\section{Carbon metabolic pathway}

Predicted metabolic pathways in the strain WiKim0068 were associated with diverse phosphotransferase (PTS) systems or permeases that transport various carbohydrates, including d-glucose, d-galactose, $d$ mannose, trehalose, sucrose, cellobiose, $\mathrm{N}$-acetyl-glucosamine, fructose, maltose, mannitol, galactitol, and lactose. The presence of these transport genes suggested that the strain WiKim0068 uses various carbohydrates for fermentation (Fig. 4). Based on the metabolic pathways, it was confirmed that the strain WiKim0068 had heterofermentative pathways.

Hexoses (glucose, fructose, and mannose) are converted to lactate, ethanol, and carbon dioxide. Additionally, $\mathrm{d}$ - and I-Lactate are produced from the reduction of pyruvate by d-lactate dehydrogenase ( $\mathrm{d}$ LDH) (EC 1.1.1.28) and I-lactate dehydrogenase (I-LDH) (EC 1.1.1.27), respectively. However, strain WiKim0068 harbors only I-LDH (locus tag: CMV25_RS07125). Notably, as shown in a previous report, ILDH was identified in Lactococcus lactis, which belongs to the same genus as the strain WiKim0068 [19]. Since d-lactate produced by LAB may induce d-lactate acidosis in some individuals [20], it is important to develop LAB for the production of dairy products that produce only l-lactate. Therefore, the lack of d-LDH is an advantage that makes the strain WiKim0068 suitable for potential applications in the dairy industry.

\section{Antibiotics resistance}


Recently, interest in foods as mediators of antibiotics resistance has been increasing. LAB, which are widely used in probiotics and as starter cultures, have the potential to serve as hosts for antibiotic resistance genes, and present the risk of transferring genes from various LAB and bacterial pathogens [21]. The strain WiKim0068 showed susceptibility to ampicillin, chloramphenicol, ciprofloxacin, erythromycin, gentamicin, penicillin, rifampin, tetracycline, and vancomycin (Table 2). Although the strain WiKim0068 was predicted to have vancomycin resistant genes in the genome, antibiotics test confirmed that it was sensitive to vancomycin.

\section{Amino acid and nicotinate and nicotinamide metabolism}

The amino acid metabolism-related genes of strain WiKim0068 were annotated using the KEGG database. Among 163 genes involved in amino acid metabolism, strain WiKim0068 harbors the most genes involved in the amino acid metabolism of cysteine, methionine, alanine, aspartate, and glutamate (Fig. 5), suggesting that the strain biosynthesize and utilize various amino acids.

Vitamin B3, one of the eight B-vitamins, is also known as nicotinate or niacin. This endogenous metabolite is an effective antioxidant that prevents oxidative damage [22]. In general, nicotinamide and nicotinate metabolites are frequently reported in Lactobacillus strains [23-25], while Lactococcus members were not known to produce these metabolites until now. Interestingly, in silico analysis of WiKim0068 genome predicted an almost complete complement metabolic pathway from the genes involved in the metabolism of nicotinate and nicotinamide (Fig. 6). Demonstrating this, $0.932 \mathrm{mg} \mathrm{L}^{-1}$ vitamin B3 was extracted from the cultured cells (Supplementary Fig. S3). These results indicated that nicotinate and nicotinamide metabolism occurs in strain WiKim0068. For comparison, studied $15 \mathrm{LAB}$ isolated from kimchi; Leuconostoc spp. produced 0.837-1.05 $\mathrm{mg} \mathrm{L}^{-1}$ vitamin $\mathrm{B} 3$, and Lactobacillus species, L. sakei, and L. curvatus produced 0.05-0.1 $\mathrm{mg} \mathrm{L}^{-1}$.

\section{Conclusions}

The complete genome of $L$. raffinolactis WiKim0068 revealed its antibiotic susceptibility, antibiotic resistance-related proteins, and its ability to produce and utilize nicotinate and nicotinamide. In addition, in vitro analysis indicated that the strain possesses beneficial health effects such as vitamin B3 production. These results suggest that $L$. raffinolactis WiKim0068 could be utilized in comparative genome analysis with other Lactococcus strains and in industrial applications.

\section{Methods}

\section{Isolation and characterization of the bacterial strain}

The strain WiKim0068 was isolated from kimchi, a Korean fermented food, in Gwangju, Korea using the dilution plating method, and incubated on De Man, Rogosa and Sharpe (MRS) agar (MB cell, LA, USA) at $30^{\circ} \mathrm{C}$ for $48 \mathrm{~h}$ under anaerobic conditions (BD GasPak ${ }^{\mathrm{TM}} \mathrm{EZ}$ Anaerobe Container Systems, New Jersey, USA). Physiological characteristics (acid production, carbon-source utilization, enzyme activity, and 
biochemical feature) were determined using the API 50CH, API ZYM, and API 20E galleries (bioMérieux, France), according to the manufacturer's instructions $[26,27]$, while the bacteria were incubated at $30^{\circ} \mathrm{C}$ for $48 \mathrm{~h}$ under anaerobic conditions. Anaerobic conditions were maintained using mineral oil.

\section{Genome sequencing and annotation}

Genomic DNA extraction was performed using the QIAcube system with a QIAamp DNeasy Blood \& Tissue Kit (Qiagen, Hilden, Germany). The genome was sequenced using the PacBio RS II sequencing system (Pacific Biosciences, Menlo Park, CA). The reads were assembled de novo using Hierarchical Genome Assembly Process version 3.0 (HGAP 3.0) in PacBio SMRT analysis version 2.3.0., as described by Jang et al. [28]. The complete genome sequence was annotated using the combined results of the automatic National Center for Biotechnology Information (NCBI) Prokaryotic Genomes Annotation Pipeline 4.1 [29] and the RAST server [30]. Phylogenetic tree based on 16S rRNA gene sequences extracted from the genome, were constructed, as described by Ismaeil et al. [31], using the neighborjoining [32], minimum-evolution [33], and maximum likelihood [34] methods, based on 1,000 randomly generated trees. Protein functions were grouped according to COG using WebMGA on-line tools (for carbohydrate metabolism, antibiotic resistance-related genes, adhesion, proteolytic enzymes, and amino acid metabolism) [35]. Nicotinate and nicotinamide metabolic pathway was mapped using the Kyoto Encyclopedia of Genes and Genomes (KEGG) [36]. The fermentative metabolic pathways were constructed based on predicted KEGG pathways and BLASTP analysis using reference gene sequences. Antimicrobial resistance genes were identified using ResFinder 3.0, available from the Center for Genomic Epidemiology (http://genomicepidemiology.org/). Prophage identification was performed using the PHAge Search Tool (PHAST) [37]. The complete genome sequences have been deposited to the DNA databank of Japan/the European Molecular Biology Laboratory/GenBank under the accession numbers CP023392-CP023394.

\section{Carbon metabolic pathway}

The fermentative metabolic pathways of $L$. raffinolactis WiKim0068 were constructed based on predicted KEGG pathways and BLASTP analysis. In detail, the genes of L. raffinolactis WiKim0068 were mapped to the five KEGG pathways (pentose phosphate pathway, fructose and mannose metabolism, pathways for pyruvate, galactose, starch, and sucrose metabolism). Then, only mapped genes were used to draw one pathway (Fig. 4), and the functions of the individual genes were reconfirmed using BLASTP.

\section{Comparative genomic analysis}

For comparative genomic analysis of strain WiKim0068, the genome sequences of two other Lactococcus raffinolactis strains: L. raffinolactis 4877 (CALL00000000.1) and L. raffinolactis NBRC $100932^{\top}$ (BCVN00000000.1) were obtained from GenBank and used as references. To determine the similarity between genome sequences, OrthoANI values of $L$. raffinolactis WiKim0068 and related strains in the genus Lactococcus were calculated using the orthologous average nucleotide identity tool (OAT software, www.ezbiocloud.net/sw/oat; ChunLab) [38]. Circular comparison map of the genomic 
sequences was created using Blast Ring Image Generator (BRIG) software [39]. Clustered regularly interspaced short palindromic repeats (CRISPRs) were analyzed using CRISPRFinder [40]. When the algorithm was detected exactly three identical (repeated and sequential) repeating regions separated by a variable order, it was considered "confirmed CRISPR".

\section{In vitro analyses}

\section{Antibiotic susceptibility test}

Antibiotic susceptibility was determined by the agar disk diffusion method on MRS agar according to the Clinical and Laboratory Standards Institute (CLSI) guidelines [41]. The $100 \mu \mathrm{L}$ inoculum $\left(10^{7}-10^{8} \mathrm{CFI}\right.$ $\mathrm{mL}^{-1}$ ) was spread on MRS plates. Antibiotic disks (Becton Dickinson Microbiology Systems, USA) were placed on MRS agar plates, incubated at $30^{\circ} \mathrm{C}$ for $48 \mathrm{~h}$, and the diameter of each clear zone was measured in millimeters. Disks containing ampicillin $(10 \mu \mathrm{g})$, chloramphenicol $(30 \mu \mathrm{g})$, ciprofloxacin (5 $\mu \mathrm{g})$, erythromycin $(15 \mu \mathrm{g})$, gentamicin $(10 \mu \mathrm{g})$, penicillin $(10 \mathrm{U})$, rifampin $(5 \mu \mathrm{g})$, tetracycline $(30 \mu \mathrm{g})$, and vancomycin $(30 \mu \mathrm{g})$ were used.

\section{Quantitative vitamin B analysis}

The strain WiKim0068 was cultured at $30^{\circ} \mathrm{C}$ for $48 \mathrm{~h}$ in MRS broth under anaerobic conditions. The cellfree supernatant was collected using a $0.22 \mu \mathrm{m}$ syringe filter. Two microliters of the cell-free supernatant was injected into the HPLC system. Vitamin B levels were determined with a NexeraX2 HPLC (Shimadzu, Japan) equipped with an LCMS-2020 LC/MS System (Shimadzu). The compounds were separated on an Aegispak C-8 column ( $150 \mathrm{~mm} \times 2 \mathrm{~mm}, 3 \mu \mathrm{m}$; Young Jin Biochrom, Korea) at $40^{\circ} \mathrm{C}$. Mobile phase A was a $0.1 \%$ formic acid in distilled water and mobile phase $B$ was $0.1 \%$ formic acid in acetonitrile. The gradient elution was as follows: from 0 to 1 min isocratic elution with $0 \%$ of mobile phase $A$, then the mobile phase B content was increased linearly to $75 \%$ in 20 min. Finally, the isocratic elution ( $25 \%$ A and $75 \%$ B) was continued for $7 \mathrm{~min}$. Solvents were delivered at a total flow rate of $0.25 \mathrm{~mL} \mathrm{~min}^{-1}$. The re-equilibration time was $5 \mathrm{~min}$. Optimal operating conditions for LC-MS/MS analysis were applied according to the method reported by Wirkus et al. [42]. Reference vitamin B group standards with $99 \%$ purity supplied by the Sigma-Aldrich (St Louis, MO, USA) were used.

\section{Abbreviations}

CDS: Coding sequences; RAST: Rapid Annotation using Subsystem Technology; PHAST: PHAge Search

Tool; KEGG: Kyoto Encyclopedia of Genes and Genomes; orthoANI: orthologous Average Nucleotide Identity; COG: Clusters of Orthologous Genes

\section{Declarations}

\section{Ethics approval and consent to participate}


Not applicable.

\section{Consent for publication}

Not applicable.

\section{Availability of data and materials}

The complete genome sequences have been deposited to the DNA databank of Japan/the European Molecular Biology Laboratory/GenBank under the accession numbers CP023392-CP023394.

\section{Competing interests}

The authors declare no competing financial interest.

\section{Funding}

This research was supported by a grant from the World Institute of Kimchi, funded by the Ministry of Science and ICT (KE2001-2) and Korea Institute of Planning and Evaluation for Technology in Food, Agriculture, Forestry and Fisheries (IPET) through Agricultural Microbiome R\&D Program, funded by Ministry of Agriculture, Food and Rural Affairs (MAFRA) [918006-04-1-HD020].

\section{Authors' contributions}

SWR and SHL designed and coordinated all the experiments. MYJ performed cultivation, DNA extraction and purification. CSL, MYJ, and MJS performed sequencing, genome assembly, gene prediction, gene annotation, and comparative genomic analysis. CSL, MYJ, SHL, and SWR wrote the manuscript. All authors have read and approved the manuscript.

\section{Acknowledgments}

Not applicable.

\section{References}

1. Bojanic M, Rasovic, Mayrhofer S, Martinovic A, Durr K, Domig KJ. Lactococci of local origin as potential starter culturesfor traditional montenegrin cheese production. Food Technol Biotechnol. 2017;55(1):55-66; doi: 10.17113/ftb.55.01.17.4854.

2. Smit G, Smit BA, Engels WJ. Flavour formation by lactic acid bacteria and biochemical flavour profiling of cheese products. FEMS Microbiol Rev. 2005;29(3):591-610; doi: 10.1016/j.femsre.2005.04.002.

3. Ouwehand AC, Salminen S, Isolauri E. Probiotics: an overview of beneficial effects. Antonie Van Leeuwenhoek. 2002;82(1-4):279-89. 
4. Schleifer KH, Kraus J, Dvorak C, Kilpper-Balz R, Collins MD, Fisher W et al. Transfer of Streptococcus lactis and related streptococci to the genus Lactococcus gen. nov. Syst Appl Microbiol. 1985;6:18395; doi: 10.1099/00221287-131-6-1531.

5. Kimoto-Nira H, Aoki R, Mizumachi K, Sasaki K. Interaction between Lactococcus lactis and Lactococcus raffinolactis during growth in milk: Development of a new starter culture. J Dairy Sci. 2012;95(4):2176-85.

6. Cavanagh D, Fitzgerald GF, McAuliffe 0 . From field to fermentation: the origins of Lactococcus lactis and its domestication to the dairy environment. Food Microbiol. 2015;47:45-61; doi: 10.1016/j.fm.2014.11.001.

7. Bourdichon F, Casaregola S, Farrokh C, Frisvad JC, Gerds ML, Hammes WP et al. Food fermentations: microorganisms with technological beneficial use. Int J Food Microbiol. 2012;154:87-97.

8. Blaiotta G, Pepe O, Mauriello G, Villani F, Andolfi R, Moschetti G. 16S-23S rDNA intergenic spacer region polymorphism of Lactococcus garvieae, Lactococcus raffinolactis and Lactococcus lactis as revealed by PCR and nucleotide sequence analysis. Syst Appl Microbiol. 2002;25(4):520-7.

9. Meslier V, Loux V, Renault P. Genome sequence of Lactococcus raffinolactis strain 4877, isolated from natural dairy starter culture. J Bacteriol. 2012;194:6364.

10. Kimoto-Nira H, Aoki R, Mizumachi K, Sasaki K, Naito H, Sawada T et al. Interaction between Lactococcus lactis and Lactococcus raffinolactis during growth in milk: development of a new starter culture. J Dairy Sci. 2012;95(4):2176-85

11. Boucher I, Vadeboncoeur C, Moineau S. Characterization of genes involved in the metabolism of agalactosides by Lactococcus raffinolactis. Appl Environ Microbiol. 2003;69(7):4049-56

12. Yan Yang S, Zheng Y, Huang Z, Min Wang X, Yang H. Lactococcus nasutitermitis sp. nov. isolated from a termite gut. Int J Syst Evol Microbiol. 2016;66(1):518-22; doi: 10.1099/ijsem.0.000743.

13. Intakes loMSCotSEoDR: Dietary reference intakes for thiamin, riboflavin, niacin, vitamin B6, folate, vitamin B12, pantothenic acid, biotin, and choline: National Academies Press (US); 1998.

14. Dobson A, Cotter PD, Ross RP, Hill C. Bacteriocin production: a probiotic trait? Appl Environ Microbiol. 2012;78(1):1-6.

15. Rouse S, Sun F, Vaughan A, Van Sinderen D. High-throughput isolation of bacteriocin-producing lactic acid bacteria, with potential application in the brewing industry. J Inst Brew. 2007;113(3):25662.

16. López-Cuellar MdR, Rodriguez-Hernandez A-I, Chavarria-Hernandez N. LAB bacteriocin applications in the last decade. Biotechnol Biotechnol Equip. 2016;30(6):1039-50.

17. LeBlanc J, Laiño JE, del Valle MJ, Vannini Vv, van Sinderen D, Taranto MP et al. B-Group vitamin production by lactic acid bacteria-current knowledge and potential applications. J Appl Microbiol. 2011;111(6):1297-309.

18. Goris J, Konstantinidis KT, Klappenbach JA, Coenye T, Vandamme P, Tiedje JM. DNA-DNA hybridization values and their relationship to whole-genome sequence similarities. Int J Syst Evol Microbiol. 2007;57(Pt 1):81-91; doi: 10.1099/ijs.0.64483-0. 
19. Guillot A, Gitton C, Anglade P, Mistou MY. Proteomic analysis of Lactococcus lactis, a lactic acid bacterium. Proteomics. 2003;3(3):337-54.

20. Mayeur C, Gratadoux J-J, Bridonneau C, Chegdani F, Larroque B, Kapel N et al. Faecal D/L lactate ratio is a metabolic signature of microbiota imbalance in patients with short bowel syndrome. PLoS One. 2013;8(1):e54335.

21. Mathur S, Singh R. Antibiotic resistance in food lactic acid bacteria-a review. Int J Food Microbiol. 2005;105(3):281-95; doi: 10.1016/j.ijfoodmicro.2005.03.008.

22. Lin Y-S, Chen S-H, Huang W-J, Chen C-H, Chien M-Y, Lin S-Y et al. Effects of nicotinic acid derivatives on tyrosinase inhibitory and antioxidant activities. Food Chem. 2012;132(4):2074-80.

23. Altermann E, Russell WM, Azcarate-Peril MA, Barrangou R, Buck BL, McAuliffe $O$ et al. Complete genome sequence of the probiotic lactic acid bacterium Lactobacillus acidophilus NCFM. Proc Natl Acad Sci U S A. 2005;102(11):3906-12.

24. Azcarate-Peril MA, Altermann E, Goh YJ, Tallon R, Sanozky-Dawes RB, Pfeiler EA et al. Analysis of the genome sequence of Lactobacillus gasseri ATCC 33323 reveals the molecular basis of an autochthonous intestinal organism. Appl Environ Microbiol. 2008;74(15):4610-25.

25. Singh P, Chung H-J, Lee I-A, D'Souza R, Kim H-J, Hong S-T. Elucidation of the anti-hyperammonemic mechanism of Lactobacillus amylovorus JBD401 by comparative genomic analysis. BMC Genomics. 2018;19(1):292.

26. Nam Y, Kim J-H, Konkit M, Kim W. Hepatoprotective effects of Lactococcus chungangensis CAU 1447 in alcoholic liver disease. J Dairy Sci. 2019;102(12):10737-47.

27. Liou J-S, Huang C-H, Wang C-L, Lee A-Y, Mori K, Tamura T et al. Lactobacillus suantsaii sp. nov., isolated from suan-tsai, a traditional Taiwanese fermented mustard green. Int J Syst Evol Microbiol. 2019;69(5):1484-9

28. Jang J-Y, Oh YJ, Lim SK, Park HK, Lee C, Kim JY et al. Salicibibacter kimchii gen. nov., sp. nov., a moderately halophilic and alkalitolerant bacterium in the family Bacillaceae, isolated from kimchi. $J$ Microbiol. 2018;56(12):880-5.

29. Tatusova T, DiCuccio M, Badretdin A, Chetvernin V, Nawrocki EP, Zaslavsky L et al. NCBI prokaryotic genome annotation pipeline. Nucleic Acids Res. 2016;44(14):6614-24; doi: 10.1093/nar/gkw569.

30. Aziz RK, Bartels D, Best AA, DeJongh M, Disz T, Edwards RA et al. The RAST Server: rapid annotations using subsystems technology. BMC Genomics. 2008;9:75; doi: 10.1186/1471-2164-9-75.

31. Ismaeil M, Yoshida N, Katayama A. Bacteroides sedimenti sp. nov., isolated from a chloroethenesdechlorinating consortium enriched from river sediment. J Microbiol. 2018;56(9):619-27.

32. Saitou N, Nei M. The neighbor-joining method: a new method for reconstructing phylogenetic trees. Mol Biol Evol. 1987;4(4):406-25.

33. Rzhetsky A, Nei M. A simple method for estimating and testing minimum-evolution trees. Mol Biol Evol. 1992;9(5):945-67. 
34. Felsenstein J. Evolutionary trees from DNA sequences: a maximum likelihood approach. J Mol Evol. 1981;17(6):368-76.

35. Wu S, Zhu Z, Fu L, Niu B, Li W. WebMGA: a customizable web server for fast metagenomic sequence analysis. BMC Genomics. 2011;12:444; doi: 10.1186/1471-2164-12-444.

36. Kanehisa M, Goto S, Sato Y, Kawashima M, Furumichi M, Tanabe M. Data, information, knowledge and principle: back to metabolism in KEGG. Nucleic Acids Res. 2014;42(Database issue):D199-205; doi: 10.1093/nar/gkt1076.

37. Zhou Y, Liang Y, Lynch KH, Dennis JJ, Wishart DS. PHAST: a fast phage search tool. Nucleic Acids Res. 2011;39(Web Server issue):W347-52; doi: 10.1093/nar/gkr485.

38. Lee I, Kim YO, Park SC, Chun J. OrthoANI: An improved algorithm and software for calculating average nucleotide identity. Int J Syst Evol Microbiol. 2015;66:1100-3; doi: 10.1099/ijsem.0.000760.

39. Alikhan NF, Petty NK, Ben Zakour NL, Beatson SA. BLAST Ring Image Generator (BRIG): simple prokaryote genome comparisons. BMC Genomics. 2011;12:402; doi: 10.1186/1471-2164-12-402.

40. Grissa I, Vergnaud G, Pourcel C. CRISPRFinder: a web tool to identify clustered regularly interspaced short palindromic repeats. Nucleic Acids Res. 2007;35(Web Server issue):W52-7; doi: 10.1093/nar/gkm360.

41. Wikler MA: Performance standards for antimicrobial susceptibility testing: Seventeenth informational supplement: Clinical and Laboratory Standards Institute; 2007.

42. Wirkus D, Jakubus A, Owczuk R, Stepnowski P, Paszkiewicz M. Development and application of novelty pretreatment method for the concurrent quantitation of eleven water-soluble $B$ vitamins in ultrafiltrates after renal replacement therapy. J Chromatogr B Analyt Technol Biomed Life Sci. 2017;1043:228-34; doi: 10.1016/j.jchromb.2016.09.021.

\section{Table}

Table 1. Comparative genomic features of Lactococcus raffinolactis WiKim0068, $L$. raffinolactis 4877, and L. raffinolactis NBRC $100932^{\mathrm{T}}$.

\begin{tabular}{llll}
\hline & \multicolumn{2}{l}{ Strain } & \\
\cline { 2 - 4 } & WiKim0068 & 4877 & NBRC 100932 \\
\hline Assembly size (Mb) & 2.29 & 2.28 & 2.18 \\
DNA G+C content (mol\%) & 39.7 & 38.7 & 39.8 \\
CDSs* & 2,187 & 2,362 & 2,123 \\
Scaffolds & 3 & 127 & 114 \\
Genes & 2,258 & 2,409 & 2,141 \\
Proteins & 2,123 & 2,070 & 2,030 \\
rRNAs & 13 & 12 & 2 \\
tRNAs & 55 & 48 & 29 \\
Finishing quality & Complete & Scaffold & Contig \\
\hline
\end{tabular}


*CDSs, Coding Sequences

Table 2. Antibiotic susceptibility of Lactococcus raffinolactis WiKim0068.

\begin{tabular}{llll}
\hline \multirow{2}{*}{ Antibiotic } & \multicolumn{3}{l}{ Antibiotic susceptibility test } \\
\cline { 2 - 5 } & Amount & Inhibition zone diameter (mm) & Resistant/Susceptible \\
\hline Ampicillin & $10 \mu \mathrm{g}$ & 30 & Susceptible \\
Chloramphenicol & $30 \mu \mathrm{g}$ & 40 & Susceptible \\
Ciprofloxacin & $5 \mu \mathrm{g}$ & 28 & Susceptible \\
Erythromycin & $15 \mu \mathrm{g}$ & 48 & Susceptible \\
Gentamicin & $10 \mu \mathrm{g}$ & 18 & Susceptible \\
Penicillin & $10 \mathrm{U}$ & 64 & Susceptible \\
Rifampin & $5 \mu \mathrm{g}$ & 42 & Susceptible \\
Tetracycline & $30 \mu \mathrm{g}$ & 65 & Susceptible \\
Vancomycin & $30 \mu \mathrm{g}$ & 23 & Susceptible \\
\hline
\end{tabular}

\section{Figures}

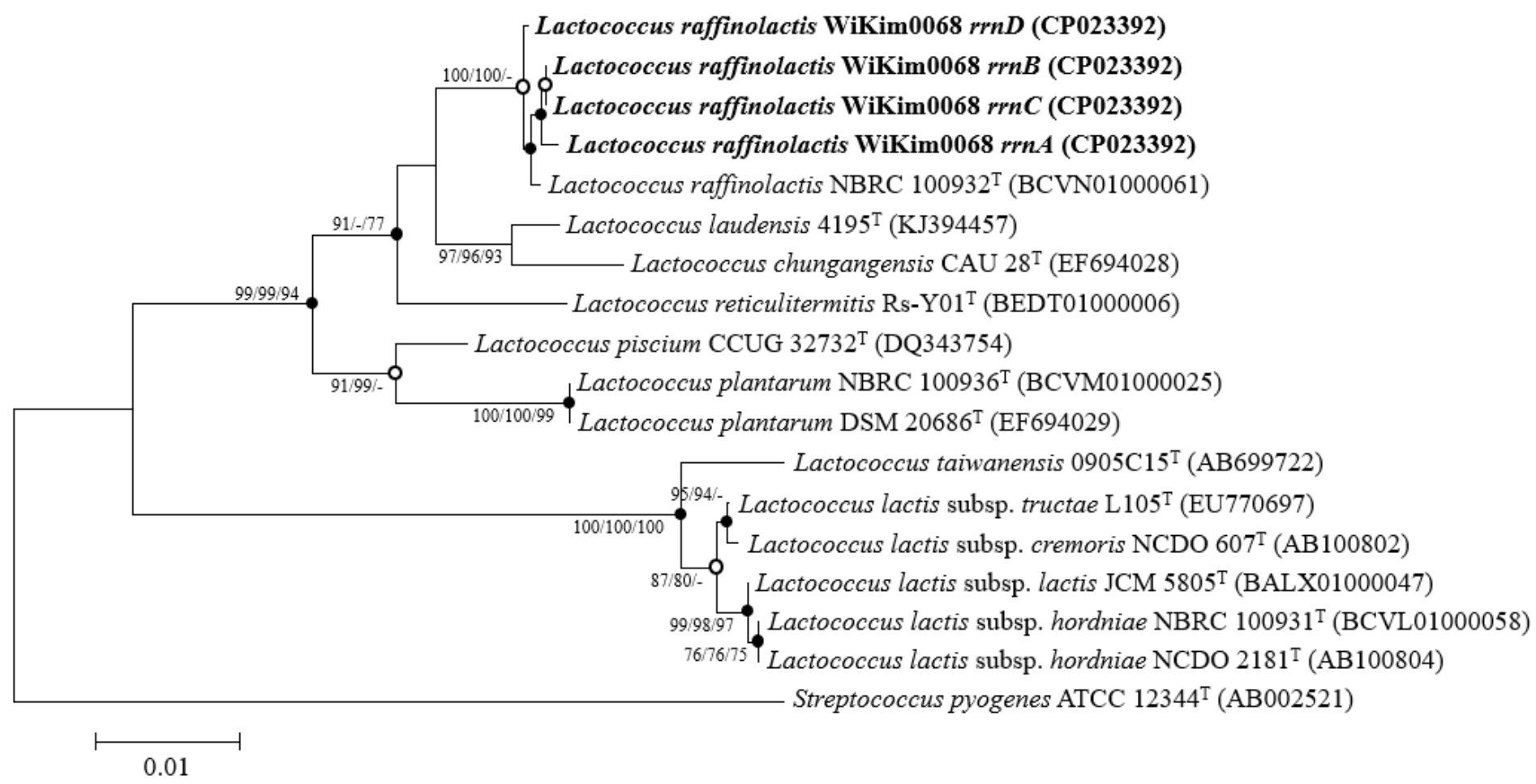


Figure 1

Phylogenetic tree based on 16S rRNA gene sequences showing the taxonomic position of the strain WiKim0068. Numbers at the nodes represent bootstrap values ( $>70 \%)$ and were calculated using neighbor-joining/minimum-evolution/maximum likelihood probabilities based on 1,000 replicates. Streptococcus pyogenes ATCC 12344 T was used as an out-group. Bar, 0.01 accumulated changes per nucleotides.

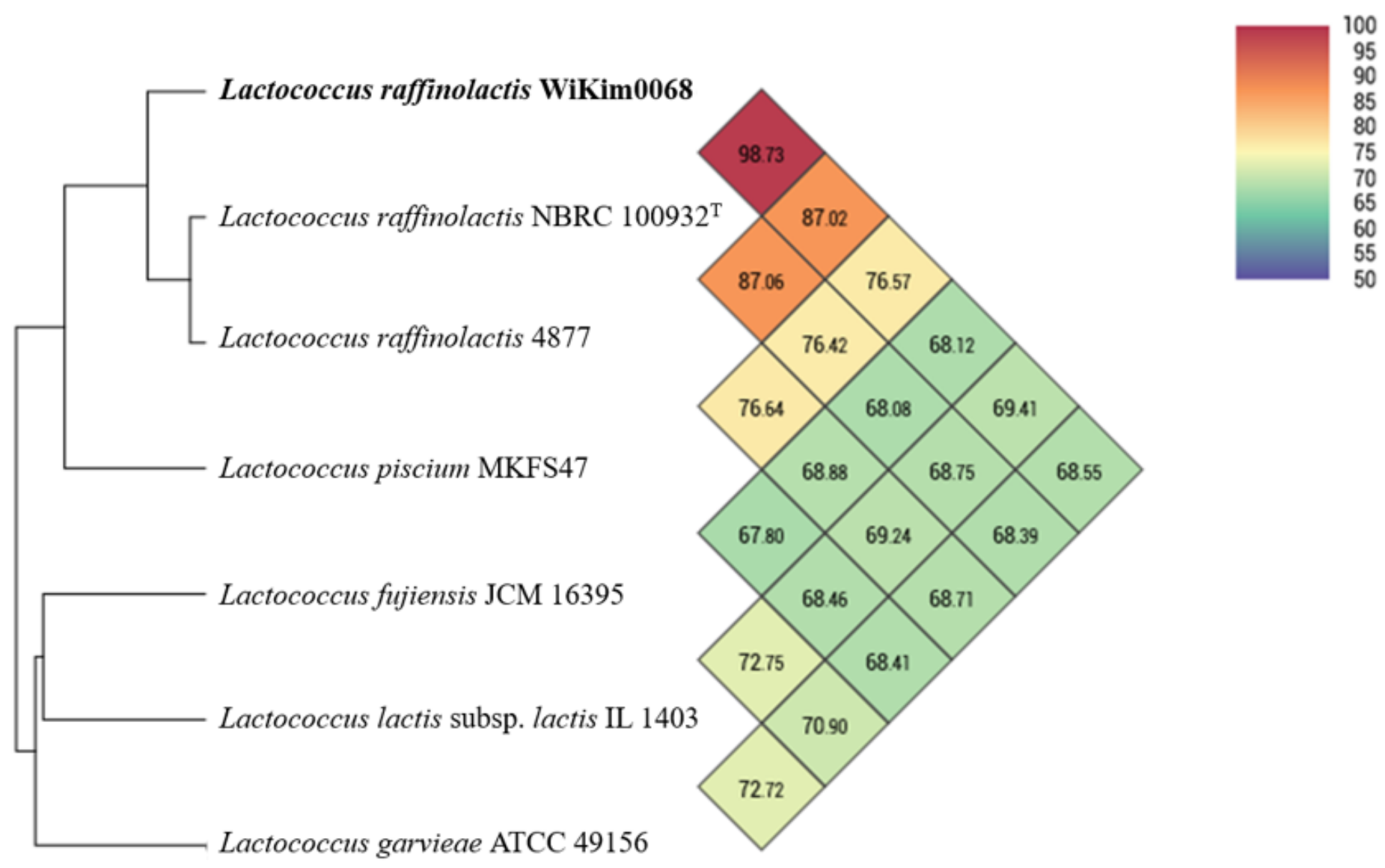

\section{Figure 2}

OrthoANI values between Lactococcus raffinolactis WiKim0068 and the closely related strains: L. raffinolactis NBRC 100932T (98.73\%), L. raffinolactis 4877 (87.02\%), L. piscium MKFS47 (76.57\%), L. fujiensis JCM 16395 (68.12\%), L. lacitis subsp. lactis IL 1403 (69.41\%), and L. garvieae ATCC 49156 (68.55\%). The orthoANI values represent the similarity between the genomes. 


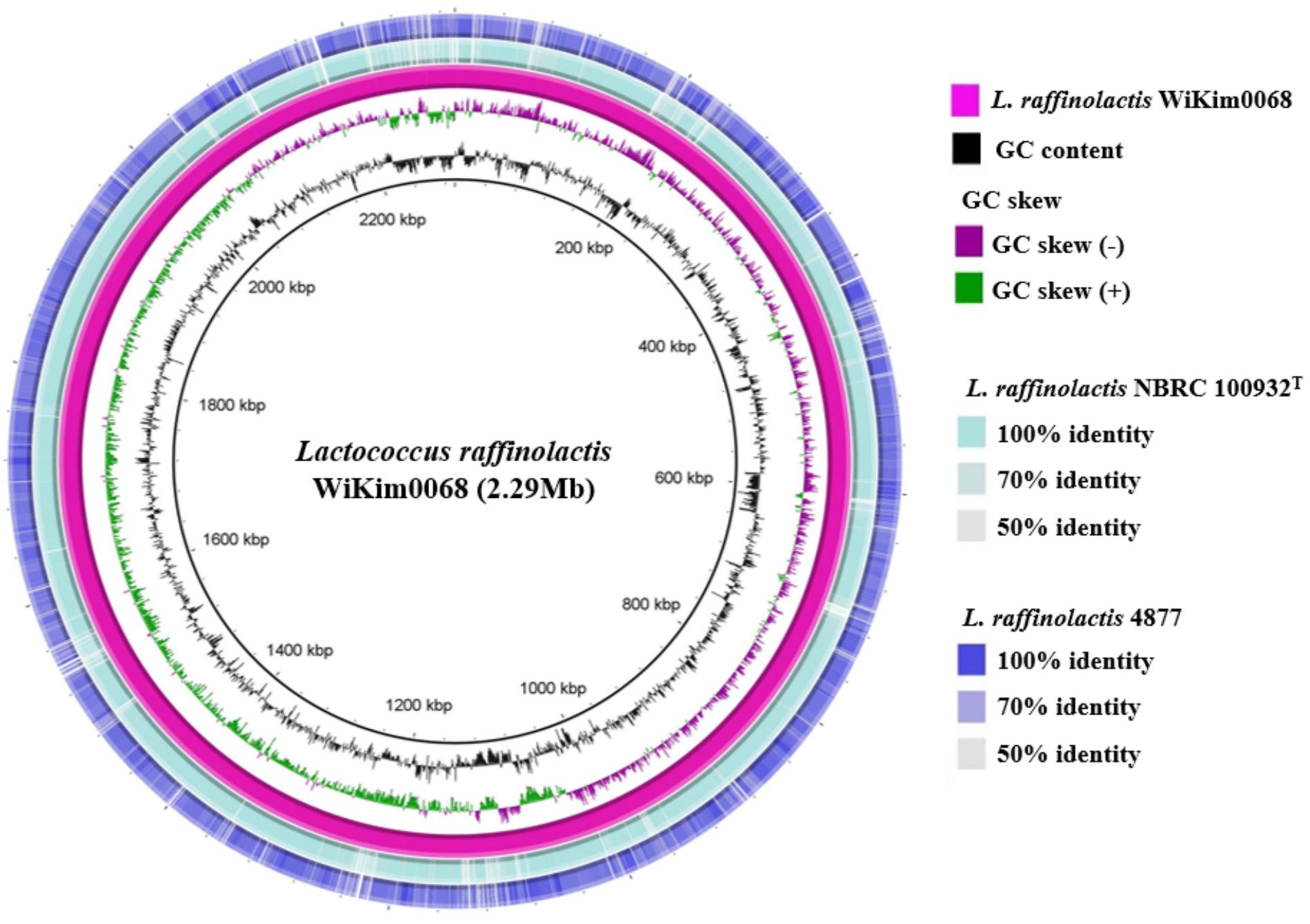

Figure 3

Circular comparison of the genomes of Lactococcus raffinolactis WiKim0068 and reference strains, L. raffinolactis NBRC $100932 T$ and L. raffinolactis 4877 . The degree of similarity between the strains is represented by color intensity. 


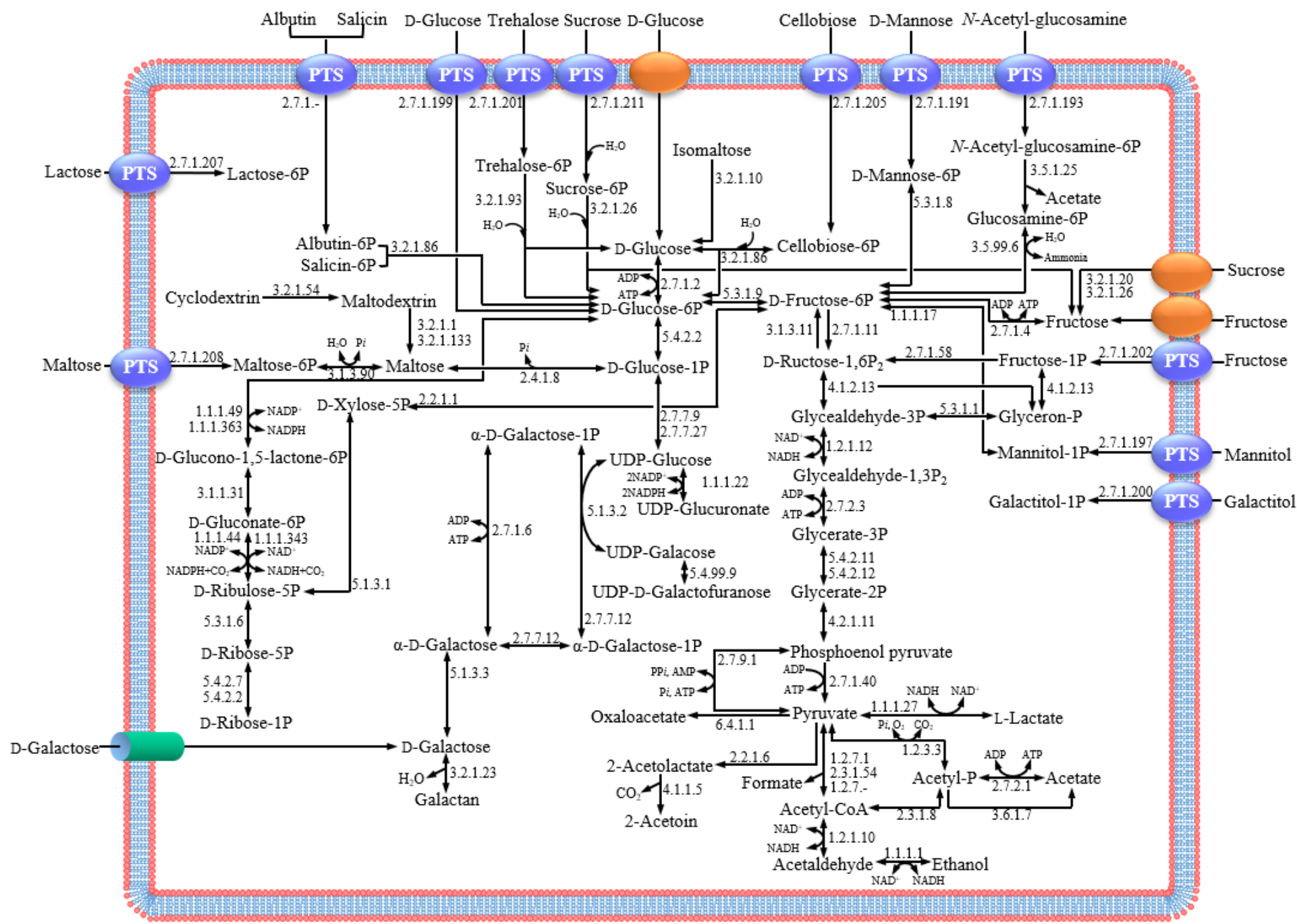

Unidentified transporting system PIS Phospho transferase system Permease

\section{Figure 4}

Predicted fermentative metabolic pathways of various carbon compounds in Lactococcus raffinolactis WiKim0068 during fermentation. PTS, phosphotransferase systems; UDP, uridine diphosphate. 
Amino acid metabolism

Metabolism of other amino acids
Cysteine and methionine metabolism Alanine, aspartate and glutamate metabolism Phenylalanine, tyrosine and tryptophan biosynthesis Glycine, serine and threonine metabolism Lysine biosynthesis

Valine, leucine and isoleucine biosynthesis Arginine biosynthesis Histidine metabolism Arginine and proline metabolism Valine, leucine and isoleucine degradation Phenylalanine metabolism Tryptophan metabolism Tyrosine metabolism

Lysine degradation Selenocompound metabolism Glutathione metabolism D-Alanine metabolism D-Glutamine and D-glutamate metabolism Taurine and hypotaurine metabolism beta-Alanine metabolism D-Arginine and D-ornithine metabolism Cyanoamino acid metabolism 0

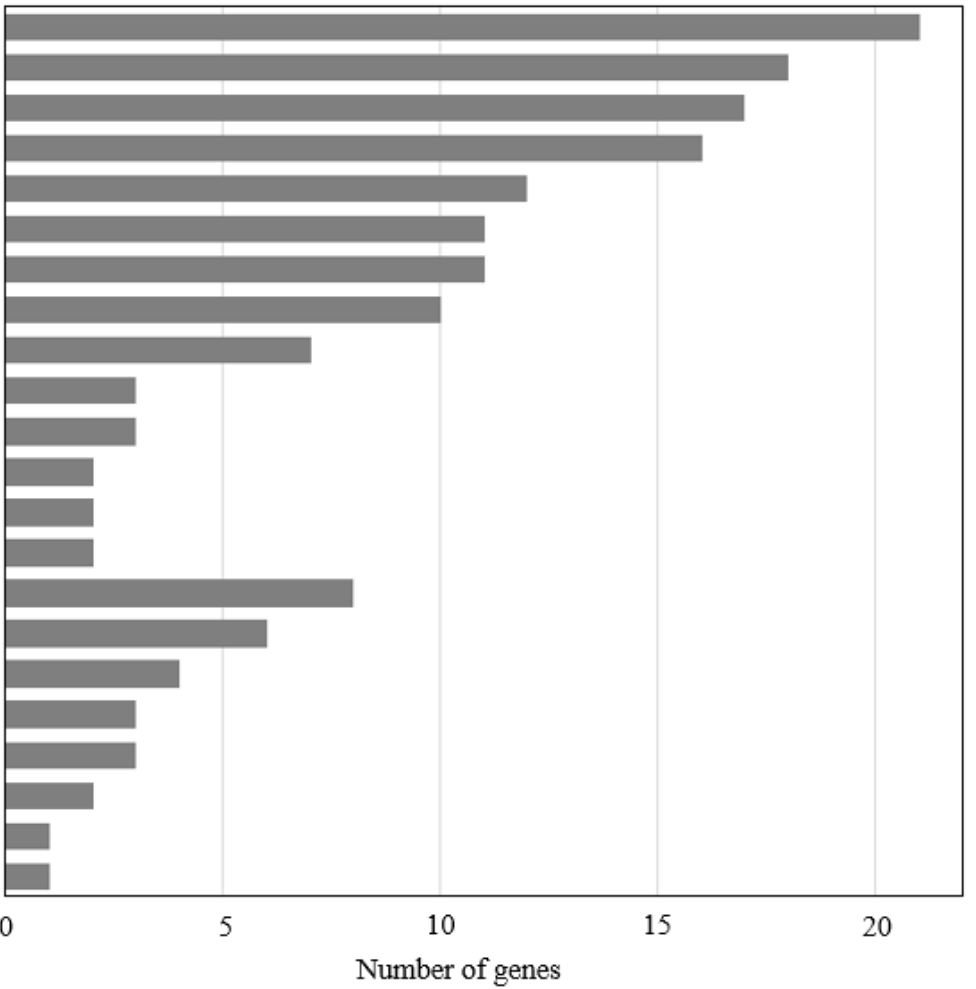

Figure 5

Amino acid metabolism-related genes of Lactococcus raffinolactis WiKim0068. 


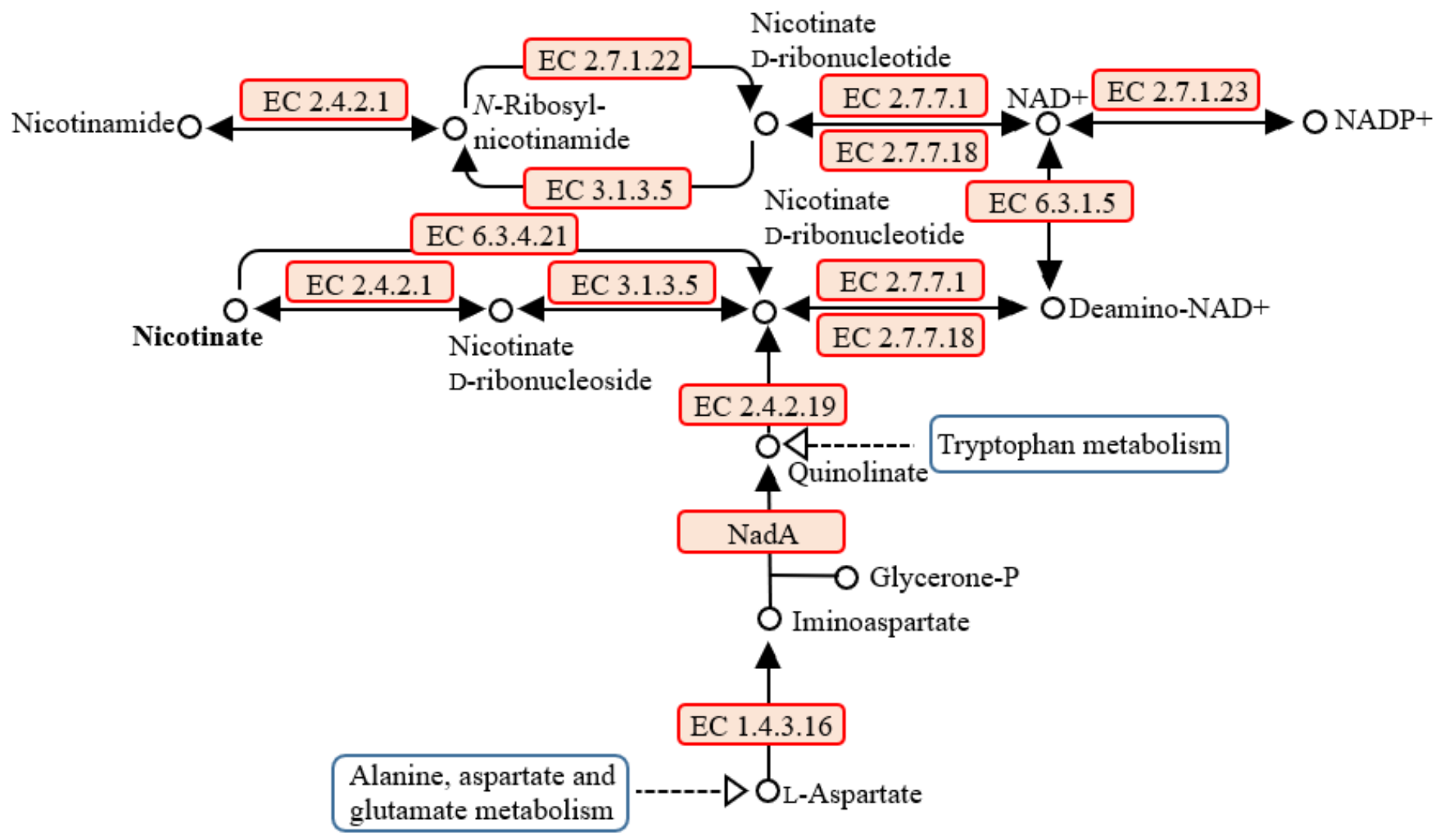

Figure 6

Nicotinate and nicotinamide metabolism in Lactococcus raffinolactis WiKim0068. Red boxes indicate enzymes in the nicotinate and nicotinamide metabolic pathway present in the strain WiKim0068.

\section{Supplementary Files}

This is a list of supplementary files associated with this preprint. Click to download.

- Supplementarydata.docx 